\title{
Comparison of the Effectiveness of Pulsed and Continuous Diclofenac Phonophoresis in Treatment of Knee Osteoarthritis
}

\author{
SAADET Deniz, MD ${ }^{1)}$, Oya Topuz, MD ${ }^{2)}$, Nilgun Simsir Atalay, MD ${ }^{2)}$, \\ AYSE SARSAN, MD ${ }^{2)}$, NeCMETTIN Yildiz, MD ${ }^{2}$, Gulin FindiKOGLU, MD ${ }^{2}$, \\ OZLEM KARACA, MD ${ }^{2)}$, FUSUN ARDIC, MD $^{2)}$ \\ 1) 13 Subat Erzincan State Hospital, Department of Physical Medicine and Rehabilitation \\ 2) Pamukkale University Medical School, Department of Physical Medicine and Rehabilitation: \\ 20210, Kinikli / Denizli, Turkey. TEL: +90 258-211-81-29, FAX: +90 258-211-81-29
}

\begin{abstract}
Purpose] The aim of this study was to compare the effectiveness of pulsed and continuous diclofenac gel phonophoresis with topical diclofenac gel treatment. [Methods] Eighty patients with knee osteoarthritis were randomly assigned to 4 groups. The first group received continuous diclofenac gel phonophoresis, the second group received pulsed diclofenac gel phonophoresis, the third group received diclofenac gel with sham ultrasound, and the fourth group received acoustic gel applied with sham ultrasound. Range of knee motion, VAS pain at rest and in activity, the WOMAC Index and $20 \mathrm{~m}$ walking time were evaluated before the treatment, at the end of treatment and 1 month after the treatment. [Results] Improvements were found in VAS pain at rest, VAS pain in activity, WOMAC pain and physical function scores in all of the groups up to 1 month after the therapy. Both modalities of phonophoresis were shown to improve VAS pain at rest, VAS pain in activity, WOMAC pain and physical function scores compared to the other treatments. There was no significant difference in the improvements between the pulsed phonophoresis group and the continuous phonophoresis group. [Conclusion] We showed that both continuous and pulsed ultrasound diclofenac gel phonophoresis is more effective for pain and functional status of patients with knee osteoarthritis than topical application of diclofenac gel, and that the effectiveness of pulsed phonophoresis and continuous phonophoresis is similar.
\end{abstract}

Key words: Phonophoresis, Diclofenac, Osteoarthritis

(This article was submitted Feb. 11, 2009, and was accepted Jul. 3, 2009)

\section{INTRODUCTION}

Osteoarthritis is the most frequently encountered rheumatological disease of the musculoskeletal system $^{1)}$, and the knee joint is one of the commonly affected joints ${ }^{2}$.

The aim of the osteoarthritis treatment is relief of pain, facilitation of joint mobility, and reduction of disability. In "EULAR 2003 recommendations", combined use of pharmacological and nonpharmacological therapeutical agents is suggested as the first line in the optimal treatment of knee osteoarthritis $^{1)}$. Oral administration of NSAID carries a risk of adverse side-effects. In studies designed with topical NSAID application, it was reported that the reliability of topical NSAID agents is high and the main adverse effect is skin reaction ${ }^{3)}$.

Phonophoresis is the name of the method which intensifies the delivery of a topical agent into underlying tissues by ultrasound ${ }^{4-6}$ ). Phonophoresis has been reported to have been used with topical application of steroids, salicylate, 
anesthetic agents, methly nicotinate, NSAID, amphotericin $B$ etc $^{6,7)}$. It is one of the many modalities for the treatment of musculoskeletal disorders such as tendinitis, tenosinovitis, temporomandibular disorders, epicondilitis and knee osteoarthritis ${ }^{8-10)}$. In the literature, there are reports on the effect of phonophoresis with different types of ultrasound, either pulsed or continuous, on drug delivery into underlying tissues ${ }^{6,11)}$. However, we could not find a study that compared the clinical outcomes of these two ultrasound modalities.

This study was designed to find out answers to 2 questions: does phonophoresis enhance the effectiveness of diclofenac gel in knee osteoarthritis, and is there any difference of effect between continuous and pulsed application of ultrasound in diclofenac gel phonophoresis?

\section{SUBJECTS AND METHODS}

The subjects were 80 volunteers, who applied to the outpatient clinic of the Department of Physical Medicine and Rehabilitation, who had been diagnosed as having knee osteoarthritis with respect to ACR criteria between December 2007 and March 2008. The design of the study was approved by the ethical committee of the Pamukkale University. All the participants were informed about the purpose, content and the protocol of the study. The subjects were selected after applying the following exclusion criteria: the existence of any surgical intervention of the lower extremity; skin lesion at the site of application; diclofenac allergy; coordination difficulty; existence of central/peripheral nervous system disorders; existence of hematological, oncological or severe cardiac diseases; or application of intra-articular injection or physical therapeutical agents within the last 1 year. Age, sex and the presence of any systemic disorders were determined by questioning and the body mass index was calculated. In addition to the medical examination, patients were screened by blood tests, (blood count, ESR, CRP, RF, liver function tests, kidney function tests) and with $\mathrm{X}$-ray findings according to the Kellgren-Lawrence Osteoarthritis Index.

This investigation was designed as a prospective, controlled and double-blinded clinical study. Patients were randomly assigned to one of 4 groups. The first group of patients received diclofenac gel phonophoresis with a continuous type of ultrasound at $1 \mathrm{MHz}$ frequency and $1.5 \mathrm{watt} / \mathrm{cm}^{2}$ intensity. The second group of patients received diclofenac gel phonophoresis with pulsed ultrasound of 1.5 watt/ $\mathrm{cm}^{2}$ intensity and $20 \%$ duty cycle. The third group received diclofenac gel with sham ultrasound, and the fourth group, the placebo group, received acoustic gel without any medication applied with sham ultrasound. A Chattonooga Group, Model 27335 ultrasound system was used for phonophoresis. All the ultrasound applications were made with a probe of $5 \mathrm{~cm}^{2}$ over $10 \mathrm{~cm}^{2}$ skin area on both the medial and lateral aspects of the knee.The duration of the phonophoresis was $5 \mathrm{~min}$ per day for 10 days. Both diclofenac and acoustic gels were purchased by our institute and the gel tubes were covered. Sessions were arranged on a non-overlapping timetable so that different groups of patients were not able to see each other. Investigators and patients were blinded to the study.

In addition, a home based exercise program including a range of motion, quadriceps strengthening and hamstring stretching was taught and prescribed as 3 sets a day, with 10 repetitions in each set. All patients were allowed to use paracetamol in case of absolute need. Patients were evaluated with VAS pain at rest and VAS pain in activity, the Western Ontario and McMaster Universities (WOMAC) Osteoarthritis Index, range of knee motion and $20 \mathrm{~m}$ walking time, three times: before the treatment, after the treatment and 1 month after the treatment.

The SPSS 10.0 statistical program was used for data analysis. Wilcoxon's test was used for intragroup comparison of parameters at different times, and Kruskall Wallis variance analysis and ANOVA with the post hoc Bonferroni correction were used for inter-group comparisons. $\mathrm{p}<0.05$ was accepted as significant.

\section{RESULTS}

There were no statistically significant differences in the distributions of age, sex, BMI (body mass index) and radiological grade of knee osteoarthritis among the groups $(p>0.05)$. The demographic parameters are given in Table 1. The measurements before the treatment, at the end of the treatment and 1 month after the treatment for each group are given in Table 2.

We found statistically significant improvements in VAS pain at rest, VAS pain in activity, WOMAC 
Table 1. Demographic characteristics of patients

\begin{tabular}{lllll}
\hline & $\begin{array}{l}\text { CP group } \\
(\mathrm{n}=20)\end{array}$ & $\begin{array}{l}\text { PP group } \\
(\mathrm{n}=20)\end{array}$ & $\begin{array}{l}\text { D group } \\
(\mathrm{n}=20)\end{array}$ & $\begin{array}{l}\text { P group } \\
(\mathrm{n}=20)\end{array}$ \\
\hline $\begin{array}{l}\text { Age } \\
(\text { mean } \pm \mathrm{SD}) \\
(\text { year })\end{array}$ & $56.40 \pm 6.51$ & $57.10 \pm 6.63$ & $54.45 \pm 6.80$ & $56.30 \pm 6.71$ \\
\hline Female/male & $17 / 3$ & $17 / 3$ & $16 / 4$ & $16 / 4$ \\
\hline $\begin{array}{l}\text { Stage of x-ray } \mathrm{n}(\%) \\
\text { Stage 2 }\end{array}$ & $3(\% 15)$ & $3(\% 15)$ & $4(\% 20)$ & $5(\% 25)$ \\
Stage 3 & $14(\% 70)$ & $13(\% 65)$ & $14(\% 70)$ & $13(\% 65)$ \\
Stage 4 & $3(\% 15)$ & $4(\% 20)$ & $2(\% 10)$ & $2(\% 10)$ \\
\hline BMI & $31.16 \pm 4.47$ & $30.14 \pm 4.29$ & $30.59 \pm 4.06$ & $29.08 \pm 3.81$ \\
\hline
\end{tabular}

CP: Continuous ultrasound diclofenac gel phonophoresis

PP: Pulsed ultrasound diclofenac gel phonophoresis

D: Sham ultrasound with diclofenac gel

P: Sham ultrasound with acoustic gel

BMI: Body mass index

Table 2. Results at three time points

\begin{tabular}{|c|c|c|c|c|c|}
\hline & & $\begin{array}{c}\text { CP group } \\
(\text { Mean } \pm \mathrm{SD})\end{array}$ & $\begin{array}{c}\text { PP group } \\
(\text { Mean } \pm \text { SD) }\end{array}$ & $\begin{array}{c}\text { D group } \\
(\text { Mean } \pm \text { SD) }\end{array}$ & $\begin{array}{c}\text { P group } \\
(\text { Mean } \pm \text { SD })\end{array}$ \\
\hline \multirow[t]{3}{*}{ VAS pain in activity } & Before treatment & $6.40 \pm 1.18$ & $6.00 \pm 1.12$ & $6.30 \pm 1.49$ & $5.75 \pm 1.44$ \\
\hline & After treatment & $3.45 \pm 1.57 * * *$ & $3.15 \pm 1.08^{* * *}$ & $4.75 \pm 1.55^{* * *}$ & $4.90 \pm 1.51^{* * *}$ \\
\hline & 1 month & $3.10 \pm 1.51^{* * *}$ & $2.60 \pm 1.31^{* * *}$ & $5.00 \pm 1.74 * *$ & $5.25 \pm 1.48^{* *}$ \\
\hline \multirow[t]{3}{*}{ VAS pain at rest } & Before treatment & $3.40 \pm 2.01$ & $3.00 \pm 1.97$ & $3.65 \pm 1.98$ & $3.40 \pm 1.75$ \\
\hline & After treatment & $1.10 \pm 1.48^{* * *}$ & $1.00 \pm 1.33^{* * *}$ & $2.60 \pm 2.06^{* *}$ & $2.65 \pm 1.66^{* *}$ \\
\hline & 1 month & $0.95 \pm 1.27 * * *$ & $0.85 \pm 1.26^{* * *}$ & $2.80 \pm 2.01^{* *}$ & $2.95 \pm 1.82 * *$ \\
\hline \multirow[t]{3}{*}{ Flexion } & Before treatment & $118.75 \pm 10.74$ & $119.00 \pm 14.38$ & $121.85 \pm 11.72$ & $121.00 \pm 11.87$ \\
\hline & After treatment & $122.00 \pm 7.84^{*}$ & $123.50 \pm 8.44^{*}$ & $122.35 \pm 11.39$ & $121.25 \pm 11.79$ \\
\hline & 1 month & $122.00 \pm 7.84^{*}$ & $124.00 \pm 7.18^{*}$ & $122.35 \pm 11.39$ & $121.25 \pm 11.79$ \\
\hline \multirow[t]{3}{*}{$20 \mathrm{~m}$ walking time } & Before treatment & $24.00 \pm 5.51$ & $22.72 \pm 5.94$ & $23.15 \pm 5.14$ & $24.55 \pm 6.85$ \\
\hline & After treatment & $21.25 \pm 4.82 * * *$ & $19.89 \pm 3.71 * * *$ & $22.15 \pm 4.24^{*}$ & $24.41 \pm 6.95$ \\
\hline & 1 month & $21.11 \pm 4.95^{* * *}$ & $19.86 \pm 3.61 * * *$ & $22.24 \pm 4.06$ & $24.56 \pm 7.06$ \\
\hline \multirow[t]{3}{*}{ WOMAC pain } & Before treatment & $15.40 \pm 3.01$ & $14.35 \pm 2.34$ & $14.80 \pm 3.01$ & $14.45 \pm 2.96$ \\
\hline & After treatment & $11.20 \pm 2.93 * * *$ & $8.95 \pm 2.23 * * *$ & $12.25 \pm 2.84 * * *$ & $13.20 \pm 3.15^{* *}$ \\
\hline & 1 month & $9.85 \pm 2.60 * *$ & $7.90 \pm 1.83 * * * *$ & $12.35 \pm 2.88^{* * *}$ & $13.80 \pm 3.25^{* *}$ \\
\hline \multirow[t]{3}{*}{ WOMAC stiffness } & Before treatment & $6.10 \pm 1.33$ & $5.55 \pm 1.63$ & $6.05 \pm 1.39$ & $5.35 \pm 1.78$ \\
\hline & After treatment & $4.60 \pm 1.60 * * *$ & $3.60 \pm 1.04 * * *$ & $5.10 \pm 1.86^{* *}$ & $5.05 \pm 1.82$ \\
\hline & 1 month & $4.40 \pm 1.46^{* *}$ & $3.40 \pm 1.23 * * *$ & $5.50 \pm 1.70^{*}$ & $5.10 \pm 1.80$ \\
\hline \multirow[t]{3}{*}{ WOMAC physical function } & Before treatment & $52.05 \pm 8.70$ & $49.85 \pm 7.00$ & $52.05 \pm 9.49$ & $47.90 \pm 9.18$ \\
\hline & After treatment & $40.50 \pm 10.27 * * *$ & $35.55 \pm 8.60 * * *$ & $46.80 \pm 9.50 * * *$ & $46.75 \pm 9.36^{* *}$ \\
\hline & 1 month & $37.15 \pm 10.49 * * *$ & $31.45 \pm 7.86^{* * *}$ & $46.80 \pm 9.24 * *$ & $47.05 \pm 9.29^{*}$ \\
\hline
\end{tabular}

CP: Continuous ultrasound diclofenac gel phonophoresis

PP: Pulsed ultrasound diclofenac gel phonophoresis

D: Sham ultrasound with diclofenac gel

P: Sham ultrasound with acoustic gel

Note: The statistical comparisons were between pre- and post-treatment measures

$* \mathrm{p}<0.05, * * \mathrm{p}<0.01, * * * \mathrm{p}<0.001$ 
pain and physical function scores for all groups at post treatment and 1 month after the treatment compared to pre-treatment values $(\mathrm{p}<0.05)$. Continuous and pulsed ultrasound phonophoresis groups were also found to have significantly improved degree of knee flexion, $20 \mathrm{~m}$ walking time in addition to the above parameters, compared to both controls after the treatment.

Sham ultrasound with diclofenac gel was found to be ineffective for degree of knee flexion at posttreatment and one month after treatment and $20 \mathrm{~m}$ walking time was found to be unchanged at one month after the treatment compared to the pretreatment state. However, this group showed significant improvements in the other parameters.

Sham ultrasound with acoustic gel did not affect $20 \mathrm{~m}$ walking time, degree of knee flexion and WOMAC stiffness scores at post-treatment and one month after the treatment, but this group showed improvements in the other parameters.

There was no significant difference in any parameter between the pulsed phonophoresis group and the continuous phonophoresis group. Both modalities of phonophoresis had significantly beter values for VAS pain at rest and VAS pain in activity, WOMAC pain and functional status indices compared to the diclofenac gel and placebo groups. The pulsed phonophoresis group had significantly better WOMAC stiffness scores than the diclofenac and placebo groups. Although the continuous phonophoresis group had better WOMAC stiffness scores than the diclofenac and placebo groups, the difference was not statistically significant. For all groups, the $20 \mathrm{~m}$ walking time and degree of knee flexion were not significantly different between pre- and all post-treatment evaluations.

\section{DISCUSSION}

In this study, the clinical efficacy of diclofenac gel by either continuous or pulsed phonophoresis was compared with topical application of diclofenac gel using patients with knee osteoarthritis. Both continuous and pulsed diclofenac phonophoresis treatments were demonstrated to be significantly more effective for VAS pain at rest, VAS pain in activity, WOMAC pain and physical function scores than topical diclofenac gel and the placebo, and the effectiveness of pulsed phonophoresis and continuous phonophoresis treatments were similar.
In a study by Niethard et al. ${ }^{12)}$, a 3 -week application of topical diclofenac diethylamine $(1.16 \%)$ gel had better impact on pain at rest and pain in activity and resulted in significantly better scores in WOMAC pain, stiffness and physical function indices than placebo in 237 patients with knee osteoarthritis. In another study, application of topical diclofenac solution, a control solution which contained dimethyl sulfoxide (DMSO) as a carrier media but no diclofenac, and a placebo solution were used as treatments for 248 patients with knee osteoarthritis. The topical diclofenac solution was found to be significantly more effective than the other applications for the WOMAC pain, stiffness and physical function indices, and pain during activity as well as global patient assessment ${ }^{13)}$. In a similar study by Baer et al. ${ }^{14)}$, patients with knee osteoarthritis were treated with topical diclofenac solution. Statistically significant improvements in WOMAC pain, stiffness and physical function, and patient global assessment scores were seen compared to a group treated with placebo. Contrary to the studies cited above, in our study, the patients who received topical application of diclofenac gel did not show any significant improvement compared to the placebo group in VAS pain at rest and VAS pain in activity, WOMAC pain, stiffness and physical function scores. This may be due to the fact that topical diclofenac was applied once a day and over a shorter period than in the other studies.

Cagnie et al. ${ }^{11)}$ studied 26 patients with knee osteoarthritis who required arthroscopy, assigning them to 3 groups. In the first group, phonophoresis of ketoprofen gel was applied with continuous ultrasound $\left(1.5 \mathrm{~W} / \mathrm{cm}^{2}\right)$ for $5 \mathrm{~min}$; in the second group the same treatment was given but with pulsed ultrasound with $20 \%$ duty cycle, and in the last group ketoprofen gel was applied with sham ultrasound. Biopsies of synovial tisssue taken after completion of the therapy demonstrated a higher ketoprofen level in synovial tissue in the groups which received the medication either by continuous or pulsed ultrasound phonophoresis than in the group which received ketoprofen gel with sham ultrasound. Additionally, concentration of ketoprofen in the synovial tissue was found to be higher in the pulsed ultrasound group than in the continuous ultrasound phonophoresis group but the result was not statistically significant. It has been reported that both the thermal and mechanical 
properties of ultrasound may play a role in vibrating the cells at high speed and changing the resting potential of the cell membrane by cavitation to enhance the diffusion of ketoprofen. We found in our study that topical application of diclofenac gel with phonophoresis increased the efficacy of the medication. However, application of diclofenac gel with pulsed ultrasound phonophoresis was not found to be any more effective than continuous ultrasound phonophoresis.

Serikov et al. ${ }^{15)}$ applied 5\% ibuprofen gel with phonophoresis twice a day and a 3rd dose of the same medicine topically to 20 patients with osteoarthritis. The control group, which was composed of 19 subjects, received only $5 \%$ ibuprofen gel topically. A statistically insignificant reduction of pain at rest, pain in walking and pain in palpation was recorded at the end of the study. Similar to our findings, the results of Serikov's study support the effectiveness of topical NSAID gel application with ultrasound. Shin et al. ${ }^{16)}$ designed a randomized double blinded study to compare the effectiveness of $1 \%$ indomethacin and placebo gel phonophoresis using 20 patients with temporomandibular joint pain. They detected a significant reduction in pain with pressure and VAS pain scores in the group that received $1 \%$ indomethacin gel phonophoresis. In another study, $0.8 \mathrm{w} / \mathrm{cm}^{2}$ pulsed ketoprofen phonophoresis was administered to patients with lateral/medial epicondyle enthesopathies for 10 days and it was found to be more effective than the application of pulsed ultrasound alone by means of subjective and objective measures ${ }^{8}$. In a study designed to detect the effectiveness of phonophoresis using 64 patients with shoulder pain, diclofenac phonophoresis was found to show better results in terms of pain at rest and activity ${ }^{17)}$. Contrary to the articles cited above, one study has proposed that the improvements seen after phonophoresis are similar to those achieved with ultrasound alone in terms of pain and physical function for patients with knee osteoarthritis ${ }^{18)}$. Our study differs from previous studies in that it is the first to show that phonophoresis enhances the effectiveness of topical diclofenac gel application and it is also the first study to compare the clinical effects of pulsed and continuous phonophoresis with each other using patients with knee osteoarthritis.

A limitation of our study is that we did not use ultrasound treatment alone. If this treatment had been included, it would also have been possible to demonstrate the effect of ultrasound alone, without any topical NSAID gel.

We demonstrated that diclofenac gel phonophoresis by either continuous or pulsed ultrasound is effective for pain and functional status of patients with knee osteoarthritis and is significantly more effective than topical diclofenac gel application. In addition, the effectiveness of both continuous and pulsed ultrasound phonophoresis on VAS pain in activity, VAS pain in rest, WOMAC pain, stiffness and physical function scores was similar.

\section{REFERENCES}

1) Jordan KM, Arden NK, Doherty M, et al.: EULAR Recommendations 2003: an evidence based approach to the management of knee osteoarthritis: Report of a Task Force of the Standing Committee for International Clinical Studies Including Therapeutic Trials (ESCISIT). Ann Rheum Dis, 2003, 62(12): 1145-1155.

2) Cooper C, Snow S, McAlindon TE, et al.: Risk factors for the incidence and progression of radiographic knee osteoarthritis. Arthritis Rheum, 2000, 43(5): 9951000.

3) Evans JM, McMahon AD, McGilchrist MM, et al.: Topical non-steroidal anti-inflammatory drugs and admission to hospital for upper gastrointestinal bleeding and perforation: a record linkage case control study. BMJ, 1995, 311: 22-26.

4) Guzman J: Physical Medicine \& Rehabilitation 3rd ed. Braddom RL(ed.), China: Elsevier Press, 2007, pp767-797.

5) Young S: Electrotheraphy Evidence Based Practice. 11 th ed. Kitchen S(ed.), Elsevier Churchill Livingstone Press, 2002, pp211-233.

6) Byl NN: The use of ultrasound as an enhancer for transcutaneous drug delivery: Phonophoresis. Phys Ther, 1995, 75(6): 539-553.

7) Oziomek RS, Perin DH, Herold DA, et al.: Effect of phonophoresis on serum salicylate levels. Med Sci Sports Exerc, 1991, 23(4): 397-401.

8) Cabak A, Maczewska M, Lyp M, et al.: The effectiveness of phonophoresis with ketoprofen in the treatment of epicondylopathy. Ortop Traumatol Rehabil, 2005, 7(6): 660-665.

9) Klaiman MD, Shrader JA, Danoff JV, et al.: Phonophoresis versus ultrasound in the treatment of common musculoskeletal conditions. Med Sci Sports Exerc, 1998, 30(9): 1349-1355.

10) Buescher JJ: Temporomandibular joint disorders. Am Fam Physician, 2007, 15(76): 1477-1482.

11) Cagnie B, Vinck E, Rimbaut S, et al.: Phonoporesis versus topical application of ketoprofen: comparison 
between tissue and plasma levels. Phys Ther, 2003, 83(8): 707-712.

12) Niethard FU, Gold MS, Solomon GS, et al.: Efficacy of topical diclofenac diethylamine gel in osteoarthritis of the knee. J Rheumatol, 2005, 32(12): 2384-2392.

13) Bookman AA, Williams KS, Shainhouse JZ: Effect of a diclofenac solution for relieving symptoms of primary osteoarthritis of the knee: a randomized controlled trial. CMAJ, 2004, 17; 171(4): 333-338.

14) Baer PA, Thomas LM, Shainhouse Z: Treatment of osteoarthritis of the knee with a topical diclofenac solution: a randomised controlled, 6-week trial. BMC Musculoskelet Disord, 2005, 6:44.
15) Serikov NP: Efficacy of ibuprofen (nurofen gel) ultraphonophoresis for pain relief in osteoarthrosis. Ter Arkh, 2007, 79(5): 79-81.

16) Shin SM, Choi JK: Effect of indomethacin phonophoresis on the relief of temporomandibular joint pain. Cranio, 1997, 15(4): 345-348.

17) Vlak T: Comparative study of the efficacy of ultrasound and sonophoresis in the treatment of painful shoulder syndrome. Rheumatizam, 1997, 46: 5-11.

18) Kozanoglu E, Basaran S, Guzel R: Short term efficacy of ibuprofen phonophoresis versus continuous ultrasound therapy in knee osteoarthritis. Swiss Med Wkly, 2003, 133(23-24): 333-338. 